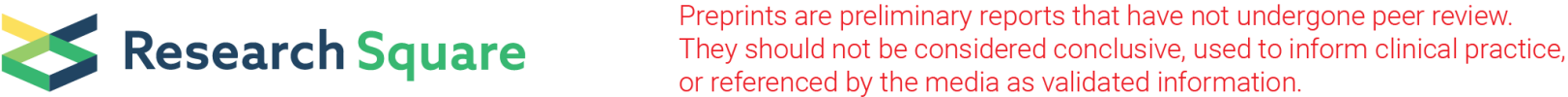

\section{Role of PVT1 Polymorphisms in the Glioma Susceptibility and Prognosis}

\author{
Xiaoying Ding \\ Xi'an Jiaotong University Second Affiliated Hospital \\ Yaqin Zhao \\ Xi'an Jiaotong University Second Affiliated Hospital \\ Haozheng Yuan \\ Xi'an Jiaotong University Second Affiliated Hospital \\ Yong Zhang \\ Xi'an Jiaotong University Second Affiliated Hospital \\ Ya Gao ( $\nabla$ xianc21lab12@163.com) \\ Xi'an Jiaotong University Second Affiliated Hospital
}

\section{Research article}

Keywords: PVT1, single-nucleotide polymorphism (SNP), glioma, susceptibility, prognosis

Posted Date: March 31st, 2020

DOI: https://doi.org/10.21203/rs.3.rs-18957/v1

License: (c) (7) This work is licensed under a Creative Commons Attribution 4.0 International License. Read Full License

Version of Record: A version of this preprint was published on January 11th, 2021. See the published version at https://doi.org/10.1097/CEJ.0000000000000636. 


\begin{abstract}
Background: Genetic factors play a crucial role in the glioma risk and prognosis of glioma patients. To explore the role of PVT1 polymorphism in the susceptibility and survival of glioma in the Chinese Han population, we conducted a case-control study.

Methods: The three single-nucleotide polymorphisms (SNPs) in PVT1 were genotyped using Agena MassARRAY from 575 patients with glioma and 500 healthy controls. We used the $\chi 2$ test to analyze the differences in distribution of allele and genotype between the cases and controls. Odds ratio (OR) and $95 \%$ confidence interval $(95 \% \mathrm{Cl})$ were calculated by logistic regression analysis to evaluate the association SNPs with glioma risk. The effects of polymorphisms and clinical features on survival of glioma patients were evaluated using the log-rank test, Kaplan-Meier and Cox regression analysis.
\end{abstract}

Results: We found that rs13255292 was associated with a decreased risk of glioma in the recessive model in overall or male; and rs4410871 was significantly associated with an increased the risk of glioma in age $\leq 40$ years old or female. Moreover, the extent of resection and chemotherapy were found to be key prognostic factors in survival of glioma patients. However, the gender, age, tumor grade, radiotherapy and PVT1 polymorphisms have no effect on prognosis of glioma patients.

Conclusions: Our results indicated that PVT1 polymorphisms (rs13255292 and rs4410871) were associated with glioma susceptibility, but have no effect on prognosis of glioma patients. Further studies with large samples are required to confirm the results.

\title{
Introduction
}

Glioma is the most common intracranial malignant tumor in the central nervous system (CNS) and has a poor prognosis and high mortality [1]. The incidence of glioma has been sharply increasing worldwide. The statistics of incidence and mortality worldwide for 36 cancers in 185 countries showed that there were 296,851 newly diagnosed cases and 241,037 individuals died from brain and CNS tumor in 2018 [2]. According to the data of National Office for Cancer Prevention and Control in China, the estimated numbers of newly brain and CNS tumor cases and deaths were 101,600 and 61,000, respectively [3]. Despite diagnosis and treatments (surgery, radiotherapy and chemotherapy) have been continuously improving, the outcomes of patients with glioma remain poor. To date, many risk factors have been identified as potential contributors to gliomas risk, such as smoking, ionizing radiation exposure, occupational exposure, environmental carcinogens, higher socioeconomic status and education level [4]. The age, gender, extent of resection, radiotherapy, chemotherapy, and histological grade, tumor size and range have been identified as potential contributors to the prognosis of glioma patients [5, 6]. Moreover, many genetic polymorphisms have been identified to be associated with the susceptibility to gliomas and as well as the prognosis of glioma patients [7-9]. Therefore, it is critical to identify new glioma therapeutic targets and new diagnostic and prognostic biomarkers.

The human plasmacytoma variant translocation 1 (PVT1) gene at the 8q24.21 chromosomal region represents a long non-coding RNA locus that has been identified as a candidate oncogene [10]. Zou et al. found that diffuse glioma patients with high PVT1 expression had poor survival outcome, aberrantly expressed PVT1 could be the independent prognosis biomarkers for glioma patients [11]. The overexpression of PVT1 increased the expression of Atg7 and Beclin 1 by targeting miR-186, which induced protective autophagy, thus promoting glioma vascular endothelial cell proliferation, migration, and angiogenesis [12]. Several polymorphisms in PVT1 have been reported to be associated with cancers risk and prognosis. The rs1561927 in PVT1 were found to be associated with poor overall survival in pancreatic ductal adenocarcinoma cases patients [13]. The GG genotype of rs13281615 in PVT1 was associated with increased risk of breast cancer likely by influencing PVT1 expression [14]. The presence of polymorphisms rs13281615 in PVT1 and rs2910164 in miR-146a contribute to a favorable prognosis in colon cancer patients by regulating COX2 expression and cell apoptosis [15].

However, the influence of PVT1 polymorphisms on the risk of glioma as well as the prognosis of glioma patients in the Chinese Han population has not been reported yet. Given the role of PVT1 in tumorigenesis and progression of glioma and prognosis of glioma patients, we hypothesized that the genetic polymorphisms in the PVT1 gene may also influence the risk of glioma and prognosis of glioma patients. To investigate this hypothesis, we recruited 575 patients with glioma and 500 healthy controls to investigate the role of the three SNPs (rs4410871, rs4733789 and rs13255292) in the PVT1 gene in glioma susceptibility and prognosis of glioma patients in the Chinese Han population.

\section{Materials And Methods Study subjects}

We randomly recruited a total of 1075 subjects, including 575 patients with glioma and 500 healthy controls from the department of Neurosurgery at Tangdu Hospital of The Fourth Military Medical University. All patients had newly diagnosed and histologically confirmed glioma by at least 2 senior neuropathologists according to the World Health Organization (WHO) classification in 2007. All controls were selected from the general health examinations in this hospital during the same period. The controls with a history of cancer or brain and central nervous system-related diseases and previously receiving radiotherapy and chemotherapy for certain diseases were excluded. All subjects were unrelated individuals of the Chinese Han.

\section{Follow-up}

The clinical follow-up of patients was performed in single-blind fashion with the end point of cardiac death. Patients were followed up through telephone calls outpatient visits, and writing communication with patients or their families by the professional medical staff every month. Overall survival (OS) was measured from the date of diagnosis with glioma to the date of death or last follow-up. Progress free survival (PFS) was calculated from the date of the pathologically confirmed to the progression of the disease, death without progression, or last clinical follow-up.

\section{Demographic and clinical data collection}

Page $2 / 14$ 
The demographic and clinical characteristics of patients with glioma were collected and regularly updated from medical records, questionnaires and followup, such as age, gender, histology types, tumor grade, surgical methods, extent of resection, treatment with radiotherapy and/or chemotherapy, date of last follow-up, status of patients (living/deceased).

\section{DNA extraction and genotyping}

The peripheral blood sample $(5 \mathrm{~mL})$ was collected from each patient with glioma and control subject into the ethylene diamine tetra-acetic acid (EDTA)containing vacutainers and stored at $-20^{\circ} \mathrm{C}$ until use. We use the GoldMag-Mini Whole Blood Genomic DNA Purification Kit (GoldMag. Co. Ltd., Xi'an, China) to extract genomic DNA of the samples according to the instructions. We performed a quality analysis of the extracted DNA by measuring its concentration and purity using a spectrophotometer (NanoDrop 2000; Thermo Fisher Scientific, Waltham, MA, USA).

The three SNPs (rs4410871, rs4733789 and rs13255292) in PVT1 were selected with minor allele frequency (MAF) $>5 \%$ in the global population from the HapMap database. The Agena Bioscience Assay Design Suite V2.0 software (https://agenacx.com/online-tools/) was used to design PCR amplification and extension primers of the three SNPs. The PVT1 polymorphisms were genotyped using the Agena MassARRAY platform with iPLEX gold chemistry (Agena Bioscience, San Diego, CA, USA) according to the protocol described. The Agena Bioscience TYPER software (version 4.0) was used to manage and analyze data.

\section{Bioinformatics analysis}

We used the online RegulomeDB software (http://regulome.stanford.edu/) and HaploReg software

(http://pubs.broadinstitute.org/mammals/haploreg/haploreg.php) to predict the possible functional effects on the PVT1 polymorphisms.

\section{Statistical analysis}

The basic descriptive statistical analysis of demographic and clinical data was conducted using SPSS 20.0 statistical package (SPSS, Chicago, IL). The Pearson's $\chi^{2}$ test and Student's t-test were used to analyze the differences in distribution of gender and age between the case and control groups, respectively. The $\chi^{2}$ test was used to examine whether the genotype frequencies of SNPs among control was consistent with Hardy-Weinberg equilibrium (HWE). The association between PVT1 polymorphisms and glioma risk was assessed under the genetic models by PLINK software (version 1.07). Odds ratios (ORs) and $95 \%$ confidence intervals (Cls) were calculated using logistic regression analysis. Kaplan-Meier method was used to evaluate the survival, and log-rank test to assess the difference between these two groups. Hazard ratio (HR) and 95\% Cl were calculated from the univariate Cox regression analysis to estimate the association between clinical factors, PVT1 polymorphisms with PFS and OS in glioma patients. Multivariate Cox models were performed to compute HR and $95 \% \mathrm{Cl}$, after adjustment potential risk factors. All $\mathrm{P}$ values were two-sided, and $\mathrm{P}<0.05$ was considered statistically significant.

\section{Results}

\section{Sample characteristics}

The basic descriptive statistics for age, gender, WHO classification, WHO grade, surgical methods, chemotherapy, radiotherapy and survival condition were computed and listed in Table 1. There were 575 patients with glioma ( 320 males and 255 females) and 500 healthy controls ( 279 males and 221 females) in this case-control study. The mean age of case and control groups was 40.46 years old and 40.53 years old, respectively. The glioma patients consisted of 353 (61.4\%) astrocytoma, 36 (6.3\%) ependymoma, 36 (6.3\%) glioblastoma, 94 (16.3\%) oligodendrocytes astrocytoma, 19 (3.3\%) oligodendroglioma and 37 (6.4\%) other gliomas. There were $369(64.2 \%)$ cases with grade I-II tumors and $206(35.8 \%)$ cases with grade III-IV tumors. $183(31.8 \%)$ patients were treated with STR or NTR; 392 (68.2\%) patients were treated with GTR. 364 (63.3\%) patients were treated with GK radiotherapy, 155 (27.0\%) patients were treated with CRT, and the rest $56(9.7 \%)$ patients did not receive any radiotherapy. 237 (41.2\%) patients were treated with chemotherapy (platinum: 118, nimustine: 70 , temozolomide: 49, respectively), while 338 (58.8\%) patients were not treated. There were 511 (88.9\%) deaths, 40 (7.0\%) patients survive, and 24 (4.2\%) patients are lost to follow-up. No statistically significant differences were found between case and control groups with regarded to age and gender distribution $(p>0.05)$. 
Table 1

Characteristics of patients with glioma and controls

\begin{tabular}{|c|c|c|c|c|}
\hline \multicolumn{2}{|l|}{ Characteristics } & Cases (\%) & Controls (\%) & $\mathbf{P}$ \\
\hline \multicolumn{2}{|l|}{ Total } & 575 & 500 & \\
\hline \multicolumn{2}{|l|}{ Age, Mean \pm SD (year) } & $40.46 \pm 18.08$ & $40.53 \pm 13.90$ & 0.942 \\
\hline \multirow[t]{2}{*}{ Gender } & Male & $320(55.7)$ & $279(55.8)$ & 0.961 \\
\hline & Female & $255(44.3)$ & $221(44.2)$ & \\
\hline \multirow[t]{6}{*}{ WHO classification } & Astrocytoma & $353(61.4)$ & & \\
\hline & Ependymoma & $36(6.3)$ & & \\
\hline & Glioblastoma & $36(6.3)$ & & \\
\hline & Oligodendrocytes astrocytoma & $94(16.3)$ & & \\
\hline & Oligodendroglioma & $19(3.3)$ & & \\
\hline & Others & $37(6.4)$ & & \\
\hline \multirow[t]{2}{*}{ WHO grade } & $I-I I$ & $369(64.2)$ & & \\
\hline & III-IV & $206(35.8)$ & & \\
\hline \multirow[t]{2}{*}{ Surgical method } & STR or NTR & $183(31.8)$ & & \\
\hline & GTR & $392(68.2)$ & & \\
\hline \multirow[t]{4}{*}{ Chemotherapy } & No & $338(58.8)$ & & \\
\hline & Platinum & $118(20.5)$ & & \\
\hline & Nimustine & $70(12.2)$ & & \\
\hline & Temozolomide & $49(8.5)$ & & \\
\hline \multirow[t]{3}{*}{ Radiotherapy } & No & $56(9.7)$ & & \\
\hline & CRT & $155(27.0)$ & & \\
\hline & GK & $364(63.3)$ & & \\
\hline \multirow[t]{3}{*}{ Survival condition } & Survival & $40(7.0)$ & & \\
\hline & Death & $511(88.9)$ & & \\
\hline & Lost & $24(4.2)$ & & \\
\hline \multicolumn{5}{|c|}{$\begin{array}{l}\text { SD: standard deviation; WHO: World Health Organization; STR: sub-total resection; NTR: near-total resection; GTR: gross-total resection; CRT: conforma } \\
\text { radiotherapy; GK: gamma knife }\end{array}$} \\
\hline
\end{tabular}

\section{PVT1 polymorphisms and glioma risk}

This study selected three SNPs in PVT1 which were successfully genotyped (call rate > 95\%). The detail information including chromosome id, position, role, allele and potential function predicted, MAF for the SNPs in cases and controls, and HWE of these variants is listed in Table 2. The genotype frequencies of the three SNPs among the controls were in agreement with the HWE $(p>0.05)$. However, three were no significant differences in the allelic frequency distribution of the three SNPs between the case group and the control group $(p>0.05)$. No significant association of the three SNPs in PVT1 with glioma risk. 
Table 2

Basic information of PVT1 polymorphisms and association with glioma risk

\begin{tabular}{|c|c|c|c|c|c|c|c|c|c|c|c|}
\hline SNP-ID & Chr & Position & Role & $\begin{array}{l}\text { Regulome } \\
\text { DB Score }\end{array}$ & Haploreg & $\begin{array}{l}\text { Allele } \\
\text { AVB }\end{array}$ & HWE-P & $\begin{array}{l}\text { MAF } \\
\text { Case }\end{array}$ & $\begin{array}{l}\text { MAF } \\
\text { Control }\end{array}$ & $\begin{array}{l}\text { OR } \\
(95 \% \\
\mathrm{Cl})\end{array}$ & $P$ \\
\hline rs4410871 & 8 & 127802783 & Intron & $2 b$ & $\begin{array}{l}\text { Promoter histone marks, enhancer } \\
\text { histone marks, DNAse, proteins } \\
\text { bound, motifs changed, selected } \\
\text { eQTL hits }\end{array}$ & $\mathrm{T} / \mathrm{C}$ & 0.690 & 0.351 & 0.339 & $\begin{array}{l}1.06 \\
(0.88- \\
1.26)\end{array}$ & 0.550 \\
\hline rs4733789 & 8 & 127822157 & Intron & 5 & $\begin{array}{l}\text { Promoter histone marks, enhancer } \\
\text { histone marks, DNAse, motifs } \\
\text { changed }\end{array}$ & $\mathrm{T} / \mathrm{C}$ & 0.418 & 0.442 & 0.451 & $\begin{array}{l}0.96 \\
(0.81- \\
1.14)\end{array}$ & 0.667 \\
\hline rs13255292 & 8 & 128064327 & Intron & 4 & $\begin{array}{l}\text { Enhancer histone marks, DNAse, } \\
\text { proteins bound, motifs changed }\end{array}$ & $\mathrm{T} / \mathrm{C}$ & 0.102 & 0.198 & 0.206 & $\begin{array}{l}0.95 \\
(0.77- \\
1.18)\end{array}$ & 0.642 \\
\hline \multicolumn{12}{|c|}{$\begin{array}{l}\text { SNP: single nucleotide polymorphism; Chr: chromosome; HWE: Hardy-Weinberg equilibrium; Regulome DB Score: } 2 \mathrm{~b} \text { : TF binding + any motif + DNase } \\
\text { Footprint + DNase peak; 4: TF binding + DNase peak; 5: TF binding or DNase peak; eQTL: expression quantitative trait loci; MAF: minor allele frequency; OR: } \\
\text { odds ratio; Cl: confidence interval. }\end{array}$} \\
\hline
\end{tabular}

To further explore the relationship between PVT1 polymorphisms and glioma risk, we performed a genetic model analysis, as shown in Table 3 . The results showed that individuals with the TT genotype of rs 13255292 was associated with a decreased risk of glioma compared with those with the CC/CT genotype in the recessive model before and after adjusted with age and gender $(\mathrm{OR}=0.53,95 \% \mathrm{Cl}: 0.29-0.99, \mathrm{p}=0.046)$. However, no any significant association was found between the SNPs (rs4410871 and rs4733789) and risk of glioma. 
Table 3

PVT1polymorphisms genotypes distribution and association with glioma risk

\begin{tabular}{|c|c|c|c|c|c|c|c|c|}
\hline SNP-ID & Model & Genotype & Case & Control & OR $(95 \% \mathrm{Cl})$ & $\mathbf{P}$ & Adjust OR (95\% Cl) & $\mathbf{P}$ \\
\hline \multirow[t]{8}{*}{ rs4410871 } & Codominant & $\mathrm{CC}$ & $249(43.3)$ & $216(43.2)$ & 1 & & 1 & \\
\hline & & CT & $248(43.1)$ & $229(45.8)$ & $0.94(0.73-1.21)$ & 0.632 & $0.94(0.73-1.21)$ & 0.636 \\
\hline & & TT & $78(13.6)$ & $55(11.0)$ & $1.23(0.83-1.82)$ & 0.298 & $1.23(0.83-1.82)$ & 0.296 \\
\hline & Dominant & $\mathrm{CC}$ & $249(43.3)$ & $216(43.2)$ & 1 & & 1 & \\
\hline & & $\mathrm{CT} / \mathrm{TT}$ & $326(56.7)$ & $284(56.8)$ & $1.00(0.78-1.27)$ & 0.973 & $1.00(0.78-1.27)$ & 0.976 \\
\hline & Recessive & $\mathrm{CC} / \mathrm{CT}$ & $497(86.4)$ & $445(89.0)$ & 1 & & 1 & \\
\hline & & TT & $78(13.6)$ & $55(11.0)$ & $1.27(0.88-1.84)$ & 0.203 & $1.27(0.88-1.84)$ & 0.202 \\
\hline & Additive & & & & $1.06(0.88-1.26)$ & 0.553 & $1.06(0.88-1.26)$ & 0.549 \\
\hline \multirow[t]{8}{*}{ rs4733789 } & Codominant & $\mathrm{CC}$ & $179(31.1)$ & $146(29.2)$ & 1 & & 1 & \\
\hline & & $\mathrm{CT}$ & $284(49.4)$ & $257(51.4)$ & $0.90(0.68-1.19)$ & 0.461 & $0.90(0.68-1.19)$ & 0.46 \\
\hline & & TT & $112(19.5)$ & $97(19.4)$ & $0.94(0.66-1.34)$ & 0.736 & $0.94(0.66-1.34)$ & 0.734 \\
\hline & Dominant & $\mathrm{CC}$ & $179(31.1)$ & $146(29.2)$ & 1 & & 1 & \\
\hline & & $\mathrm{CT} / \mathrm{TT}$ & $396(68.9)$ & $354(70.8)$ & $0.91(0.70-1.19)$ & 0.492 & $0.91(0.70-1.19)$ & 0.49 \\
\hline & Recessive & $\mathrm{CC} / \mathrm{CT}$ & $463(80.5)$ & $403(80.6)$ & 1 & & 1 & \\
\hline & & TT & $112(19.5)$ & $97(19.4)$ & $1.01(0.74-1.36)$ & 0.974 & $1.01(0.74-1.36)$ & 0.976 \\
\hline & Additive & & & & $0.96(0.81-1.14)$ & 0.664 & $0.96(0.81-1.14)$ & 0.661 \\
\hline \multirow[t]{8}{*}{ rs13255292 } & Codominant & $\mathrm{CC}$ & $364(63.4)$ & $320(64.3)$ & 1 & & 1 & \\
\hline & & CT & $193(33.6)$ & $151(30.3)$ & $1.12(0.87-1.46)$ & 0.381 & $1.13(0.87-1.46)$ & 0.378 \\
\hline & & $\mathrm{TT}$ & $17(3.0)$ & $27(5.4)$ & $0.55(0.30-1.03)$ & 0.064 & $0.55(0.30-1.04)$ & 0.064 \\
\hline & Dominant & $\mathrm{CC}$ & $364(63.4)$ & $320(64.3)$ & 1 & & 1 & \\
\hline & & $\mathrm{CT} / \mathrm{TT}$ & $210(36.6)$ & $178(35.7)$ & $1.04(0.81-1.33)$ & 0.775 & $1.04(0.81-1.33)$ & 0.773 \\
\hline & Recessive & $\mathrm{CC} / \mathrm{CT}$ & $557(97.0)$ & $471(94.6)$ & 1 & & 1 & \\
\hline & & $\mathrm{TT}$ & $17(3.0)$ & $27(5.4)$ & $0.53(0.29-0.99)$ & 0.046 & $0.53(0.29-0.99)$ & 0.046 \\
\hline & Additive & & & & $0.95(0.77-1.18)$ & 0.642 & $0.95(0.77-1.18)$ & 0.643 \\
\hline \multicolumn{9}{|c|}{ SNP: single nucleotide polymorphism; OR: odds ratio; Cl: confidence interval. } \\
\hline \multicolumn{9}{|c|}{ Adjust OR $(95 \% \mathrm{Cl})$ were calculated by logistic regression analysis with adjustments for age and gender. } \\
\hline & & ificon & & & & & & \\
\hline
\end{tabular}

In order to reduce the impact of age and gender on the results of statistical analysis, we conducted stratification analysis (Table 4). Our results found that individuals with the TT genotype of rs 4410871 was associated with an increased risk of glioma compared with those with the CC genotype in age $\leq 40$ years old $(\mathrm{OR}=2.05,95 \% \mathrm{Cl}: 1.12-3.75, \mathrm{p}=0.020)$. Meanwhile, rs 4410871 was found to be associated with an increased risk of glioma in the recessive model in age $\leq 40$ years old (TT vs. CC/CT: OR $=2.33,95 \% \mathrm{Cl}: 1.31-4.15, \mathrm{p}=0.004)$. 
Association of PVT1 polymorphisms with glioma risk stratified by age and gender

\begin{tabular}{|c|c|c|c|c|c|c|c|c|c|c|}
\hline \multirow[t]{2}{*}{ SNP_ID } & \multirow[t]{2}{*}{ Model } & \multirow[t]{2}{*}{ Genotype } & \multicolumn{4}{|l|}{ Age $>40$} & \multicolumn{4}{|l|}{ Age $\leq 40$} \\
\hline & & & Case (\%) & $\begin{array}{l}\text { Control } \\
(\%)\end{array}$ & OR (95\% Cl) & $\mathbf{P}$ & Case (\%) & $\begin{array}{l}\text { Control } \\
(\%)\end{array}$ & OR $(95 \% \mathrm{Cl})$ & $\mathbf{P}$ \\
\hline \multirow[t]{8}{*}{ rs4410871 } & Codominant & $\mathrm{CC}$ & $120(40.5)$ & $100(42.6)$ & 1 & & $129(46.2)$ & $116(43.8)$ & 1 & \\
\hline & & CT & $139(47)$ & $100(42.6)$ & $\begin{array}{l}1.17(0.81- \\
1.70)\end{array}$ & 0.410 & 109 (39.1) & $129(48.7)$ & $\begin{array}{l}0.77(0.53- \\
1.11)\end{array}$ & 0.163 \\
\hline & & $\mathrm{TT}$ & 37 (12.5) & 35 (14.9) & $\begin{array}{l}0.92(0.54- \\
1.57)\end{array}$ & 0.754 & $41(14.7)$ & $20(7.5)$ & $\begin{array}{l}2.05(1.12- \\
3.75)\end{array}$ & 0.020 \\
\hline & Dominant & $\mathrm{CC}$ & $120(40.5)$ & $100(42.6)$ & 1 & & $129(46.2)$ & $116(43.8)$ & 1 & \\
\hline & & $\mathrm{CT} / \mathrm{TT}$ & $176(59.5)$ & $135(57.4)$ & $\begin{array}{l}1.11(0.78- \\
1.57)\end{array}$ & 0.575 & $150(53.8)$ & $149(56.2)$ & $\begin{array}{l}0.94(0.66- \\
1.32)\end{array}$ & 0.705 \\
\hline & Recessive & $\mathrm{CC} / \mathrm{CT}$ & 259 (87.5) & $200(85.1)$ & 1 & & 238 (85.3) & 245 (92.5) & 1 & \\
\hline & & $\mathrm{TT}$ & $37(12.5)$ & 35 (14.9) & $\begin{array}{l}0.85(0.51- \\
1.40)\end{array}$ & 0.511 & 41 (14.7) & $20(7.5)$ & $\begin{array}{l}2.33(1.31- \\
4.15)\end{array}$ & 0.004 \\
\hline & Additive & & & & $\begin{array}{l}1.01(0.79- \\
1.30)\end{array}$ & 0.941 & & & $\begin{array}{l}1.16(0.89- \\
1.50)\end{array}$ & 0.270 \\
\hline \multirow[t]{8}{*}{ rs4733789 } & Codominant & $\mathrm{CC}$ & $101(34.1)$ & 65 (27.7) & 1 & & $78(28)$ & $81(30.6)$ & 1 & \\
\hline & & CT & $138(46.6)$ & $124(52.8)$ & $\begin{array}{l}0.72(0.48- \\
1.07)\end{array}$ & 0.100 & $146(52.3)$ & $133(50.2)$ & $\begin{array}{l}1.12(0.75- \\
1.66)\end{array}$ & 0.594 \\
\hline & & TT & $57(19.3)$ & 46 (19.6) & $\begin{array}{l}0.80(0.48- \\
1.32)\end{array}$ & 0.378 & 55 (19.7) & $51(19.2)$ & $\begin{array}{l}1.10(0.66- \\
1.82)\end{array}$ & 0.723 \\
\hline & Dominant & $\mathrm{CC}$ & $101(34.1)$ & 65 (27.7) & 1 & & $78(28)$ & 81 (30.6) & 1 & \\
\hline & & $\mathrm{CT} / \mathrm{TT}$ & 195 (65.9) & $170(72.3)$ & $\begin{array}{l}0.74(0.51- \\
1.08)\end{array}$ & 0.114 & $201(72)$ & $184(69.4)$ & $\begin{array}{l}1.11(0.76- \\
1.62)\end{array}$ & 0.591 \\
\hline & Recessive & $\mathrm{CC} / \mathrm{CT}$ & 239 (80.7) & $189(80.4)$ & 1 & & 224 (80.3) & $214(80.8)$ & 1 & \\
\hline & & TT & $57(19.3)$ & $46(19.6)$ & $\begin{array}{l}0.98(0.63- \\
1.51)\end{array}$ & 0.929 & 55 (19.7) & $51(19.2)$ & $\begin{array}{l}1.02(0.66- \\
1.58)\end{array}$ & 0.922 \\
\hline & Additive & & & & $\begin{array}{l}0.87(0.68- \\
1.11)\end{array}$ & 0.274 & & & $\begin{array}{l}1.05(0.82- \\
1.35)\end{array}$ & 0.681 \\
\hline \multirow[t]{9}{*}{ rs13255292 } & Codominant & $\mathrm{CC}$ & $187(63.4)$ & $150(63.8)$ & 1 & & 177 (63.4) & 170 (64.6) & 1 & \\
\hline & & $\mathrm{CT}$ & $96(32.5)$ & 73 (31.1) & $\begin{array}{l}1.03(0.71- \\
1.50)\end{array}$ & 0.881 & $97(34.8)$ & 78 (29.7) & $\begin{array}{l}1.30(0.89- \\
1.90)\end{array}$ & 0.168 \\
\hline & & TT & $12(4.1)$ & $12(5.1)$ & $\begin{array}{l}0.87(0.38- \\
2.00)\end{array}$ & 0.737 & $5(1.8)$ & $15(5.7)$ & $\begin{array}{l}0.39(0.14- \\
1.11)\end{array}$ & 0.076 \\
\hline & Dominant & $\mathrm{CC}$ & $187(63.4)$ & $150(63.8)$ & 1 & & $177(63.4)$ & $170(64.6)$ & 1 & \\
\hline & & $\mathrm{CT} / \mathrm{TT}$ & $108(36.6)$ & $85(36.2)$ & $\begin{array}{l}1.01(0.70- \\
1.44)\end{array}$ & 0.971 & $102(36.6)$ & $93(35.4)$ & $\begin{array}{l}1.16(0.81- \\
1.67)\end{array}$ & 0.418 \\
\hline & Recessive & $\mathrm{CC} / \mathrm{CT}$ & 283 (95.9) & 223 (94.9) & 1 & & $274(98.2)$ & 248 (94.3) & 1 & \\
\hline & & TT & $12(4.1)$ & $12(5.1)$ & $\begin{array}{l}0.86(0.38- \\
1.96)\end{array}$ & 0.718 & $5(1.8)$ & $15(5.7)$ & $0.35(0.12-1.00)$ & 0.050 \\
\hline & Additive & & & & $\begin{array}{l}0.99(0.73- \\
1.33)\end{array}$ & 0.921 & & & $\begin{array}{l}1.00(0.73- \\
1.36)\end{array}$ & 0.996 \\
\hline & & & Male & & & & Female & & & \\
\hline \multirow[t]{2}{*}{ rs4410871 } & Codominant & $\mathrm{CC}$ & $\begin{array}{l}135 \\
(42.19)\end{array}$ & $\begin{array}{l}111 \\
(39.78)\end{array}$ & 1 & & $\begin{array}{l}114 \\
(44.71)\end{array}$ & $\begin{array}{l}105 \\
(47.51)\end{array}$ & 1 & \\
\hline & & $\mathrm{CT}$ & $\begin{array}{l}142 \\
(44.38)\end{array}$ & $\begin{array}{l}130 \\
(46.59)\end{array}$ & $\begin{array}{l}0.90(0.64- \\
1.27)\end{array}$ & 0.547 & $\begin{array}{l}106 \\
(41.57)\end{array}$ & $99(44.8)$ & $\begin{array}{l}0.99(0.67- \\
1.44)\end{array}$ & 0.943 \\
\hline
\end{tabular}

SNP: single nucleotide polymorphism; OR: odds ratio; $\mathrm{Cl}$ : confidence interval.

OR $(95 \% \mathrm{Cl})$ were calculated by logistic regression analysis with adjustments for age and gender.

$p<0.05$ indicates statistical significance. 


\begin{tabular}{|c|c|c|c|c|c|c|c|c|c|c|}
\hline \multirow[t]{2}{*}{ SNP_ID } & \multirow[t]{2}{*}{ Model } & \multirow[t]{2}{*}{ Genotype } & \multicolumn{4}{|l|}{ Age $>40$} & \multicolumn{4}{|l|}{ Age $\leq 40$} \\
\hline & & & Case (\%) & $\begin{array}{l}\text { Control } \\
\text { (\%) }\end{array}$ & OR $(95 \% \mathrm{Cl})$ & $P$ & Case (\%) & $\begin{array}{l}\text { Control } \\
\text { (\%) }\end{array}$ & OR $(95 \% \mathrm{Cl})$ & $P$ \\
\hline & & TT & $43(13.44)$ & 38 (13.62) & $\begin{array}{l}0.93(0.56- \\
1.54)\end{array}$ & 0.783 & 35 (13.73) & 17 (7.69) & $1.90(1.00-3.59)$ & 0.049 \\
\hline & Dominant & $\mathrm{CC}$ & $\begin{array}{l}135 \\
(42.19)\end{array}$ & $\begin{array}{l}111 \\
(39.78)\end{array}$ & 1 & & $\begin{array}{l}114 \\
(44.71)\end{array}$ & $\begin{array}{l}105 \\
(47.51)\end{array}$ & 1 & \\
\hline & & $\mathrm{CT} / \mathrm{TT}$ & $\begin{array}{l}185 \\
(57.81)\end{array}$ & $\begin{array}{l}168 \\
(60.22)\end{array}$ & $\begin{array}{l}0.91(0.65- \\
1.26)\end{array}$ & 0.556 & $\begin{array}{l}141 \\
(55.29)\end{array}$ & $\begin{array}{l}116 \\
(52.49)\end{array}$ & $\begin{array}{l}1.12(0.78- \\
1.61)\end{array}$ & 0.540 \\
\hline & Recessive & $\mathrm{CC} / \mathrm{CT}$ & $\begin{array}{l}277 \\
(86.56)\end{array}$ & $\begin{array}{l}241 \\
(86.38)\end{array}$ & 1 & & $\begin{array}{l}220 \\
(86.27)\end{array}$ & $\begin{array}{l}204 \\
(92.31)\end{array}$ & 1 & \\
\hline & & $\mathrm{TT}$ & $43(13.44)$ & 38 (13.62) & $\begin{array}{l}0.99(0.62- \\
1.58)\end{array}$ & 0.952 & 35 (13.73) & 17 (7.69) & $\begin{array}{l}1.91(1.04- \\
3.51)\end{array}$ & 0.038 \\
\hline & Additive & & & & $\begin{array}{l}0.95(0.75- \\
1.20)\end{array}$ & 0.651 & & & $\begin{array}{l}1.22(0.93- \\
1.60)\end{array}$ & 0.151 \\
\hline \multirow[t]{8}{*}{ rs4733789 } & Codominant & $\mathrm{CC}$ & $96(30)$ & $86(30.82)$ & 1 & & $83(32.55)$ & $60(27.15)$ & 1 & \\
\hline & & CT & $\begin{array}{l}169 \\
(52.81)\end{array}$ & $\begin{array}{l}144 \\
(51.61)\end{array}$ & $\begin{array}{l}1.05(0.73- \\
1.52)\end{array}$ & 0.790 & $115(45.1)$ & $\begin{array}{l}113 \\
(51.13)\end{array}$ & $\begin{array}{l}0.74(0.48- \\
1.12)\end{array}$ & 0.154 \\
\hline & & TT & 55 (17.19) & $49(17.56)$ & $\begin{array}{l}1.01(0.62- \\
1.63)^{(0)}\end{array}$ & 0.982 & 57 (22.35) & $48(21.72)$ & $\begin{array}{l}0.86(0.52- \\
1.43)\end{array}$ & 0.555 \\
\hline & Dominant & CC & $96(30)$ & $86(30.82)$ & 1 & & $83(32.55)$ & $60(27.15)$ & 1 & \\
\hline & & $\mathrm{CT} / \mathrm{TT}$ & $224(70)$ & $\begin{array}{l}193 \\
(69.18)\end{array}$ & $\begin{array}{l}1.04(0.73- \\
1.47)\end{array}$ & 0.828 & $\begin{array}{l}172 \\
(67.45)\end{array}$ & $\begin{array}{l}161 \\
(72.85)\end{array}$ & $\begin{array}{l}0.77(0.52- \\
1.15)\end{array}$ & 0.201 \\
\hline & Recessive & $\mathrm{CC} / \mathrm{CT}$ & $\begin{array}{l}265 \\
(82.81)\end{array}$ & $\begin{array}{l}230 \\
(82.44)\end{array}$ & 1 & & $\begin{array}{l}198 \\
(77.65)\end{array}$ & $\begin{array}{l}173 \\
(78.28)\end{array}$ & 1 & \\
\hline & & TT & 55 (17.19) & $49(17.56)$ & $\begin{array}{l}0.97(0.64- \\
1.49)\end{array}$ & 0.904 & 57 (22.35) & 48 (21.72) & $\begin{array}{l}1.04(0.67- \\
1.60)\end{array}$ & 0.867 \\
\hline & Additive & & & & $\begin{array}{l}1.01(0.80- \\
1.28)\end{array}$ & 0.936 & & & $\begin{array}{l}0.91(0.71- \\
1.17)\end{array}$ & 0.470 \\
\hline \multirow[t]{8}{*}{ rs13255292 } & Codominant & $\mathrm{CC}$ & $\begin{array}{l}210 \\
(65.83)\end{array}$ & $\begin{array}{l}184 \\
(66.19)\end{array}$ & 1 & & $\begin{array}{l}154 \\
(60.39)\end{array}$ & $\begin{array}{l}136 \\
(61.82)\end{array}$ & 1 & \\
\hline & & Ст & $\begin{array}{l}100 \\
(31.35)\end{array}$ & $76(27.34)$ & $\begin{array}{l}1.16(0.81- \\
1.65)^{(1)}\end{array}$ & 0.431 & 93 (36.47) & 75 (34.09) & $\begin{array}{l}1.10(0.75- \\
1.60)\end{array}$ & 0.641 \\
\hline & & $\mathrm{TT}$ & $9(2.82)$ & $18(6.47)$ & $\begin{array}{l}0.44(0.19- \\
1.00)\end{array}$ & 0.051 & $8(3.14)$ & $9(4.09)$ & $\begin{array}{l}0.79(0.29- \\
2.09)\end{array}$ & 0.628 \\
\hline & Dominant & $\mathrm{CC}$ & $\begin{array}{l}210 \\
(65.83)\end{array}$ & $\begin{array}{l}184 \\
(66.19)\end{array}$ & 1 & & $\begin{array}{l}154 \\
(60.39)\end{array}$ & $\begin{array}{l}136 \\
(61.82)\end{array}$ & 1 & \\
\hline & & $\mathrm{CT} / \mathrm{TT}$ & $\begin{array}{l}109 \\
(34.17)\end{array}$ & $94(33.81)$ & $\begin{array}{l}1.02(0.72- \\
1.43)\end{array}$ & 0.917 & $\begin{array}{l}101 \\
(39.61)\end{array}$ & $84(38.18)$ & $\begin{array}{l}1.06(0.73- \\
1.54)\end{array}$ & 0.751 \\
\hline & Recessive & $\mathrm{CC} / \mathrm{CT}$ & $\begin{array}{l}310 \\
(97.18)\end{array}$ & $\begin{array}{l}260 \\
(93.53)\end{array}$ & 1 & & $\begin{array}{l}247 \\
(96.86)\end{array}$ & $\begin{array}{l}211 \\
(95.91)\end{array}$ & 1 & \\
\hline & & $\mathrm{TT}$ & $9(2.82)$ & $18(6.47)$ & $\begin{array}{l}0.42(0.19- \\
0.95)\end{array}$ & 0.037 & $8(3.14)$ & $9(4.09)$ & $0.76(0.29-2.00)$ & 0.578 \\
\hline & Additive & & & & $\begin{array}{l}0.91(0.68- \\
1.20)\end{array}$ & 0.489 & & & $\begin{array}{l}1.02(0.74- \\
1.40)\end{array}$ & 0.928 \\
\hline \multicolumn{11}{|c|}{ SNP: single nucleotide polymorphism; OR: odds ratio; Cl: confidence interval. } \\
\hline \multicolumn{11}{|c|}{ OR (95\% Cl) were calculated by logistic regression analysis with adjustments for age and gender. } \\
\hline$p<0.05$ indic & tes statistical & ignificance. & & & & & & & & \\
\hline
\end{tabular}

Gender stratification analysis results showed that rs4410871 was also associated with an increased risk of glioma in female after adjusted with age (TT vs. CC: $O R=1.90,95 \% \mathrm{Cl}: 1.00-3.59, p=0.049 ;$ TT vs. CC/CT: OR = 1.91, 95\% Cl: 1.04-3.51, p = 0.038). Moreover, rs 13255292 was found to be associated with a reduced risk of glioma in the recessive model in male after adjusted with age (TT vs. CC/CT: OR =0.42, 95\% Cl: 0.19-0.95, p=0.037) $(\mathrm{Table} 4)$.

\section{Clinical factors and prognosis of glioma patients}

We also investigated the impact clinical factors on the OS and PFS of glioma patients (Table 5). The univariate and Cox regression analysis results that the glioma patients with gross-total resection (GTR) was also associated with a reduced risk of death on OS (log-rank $p<0.001, \mathrm{HR}=0.63,95 \% \mathrm{Cl}$ : $0.52-0.76, \mathrm{p}<$ 
0.001 ) and PFS (log-rank $p<0.001, \mathrm{HR}=0.59,95 \% \mathrm{Cl}: 0.49-0.71, \mathrm{p}<0.001)$, compared with the glioma patients with near-total resection (NTR) or sub-total resection (STR). In addition, we also found that the glioma patients with the chemotherapy treatment had a longer OS (log-rank $p<0.001)$ and PFS (log-rank $p$ $=0.012$ ), and had a better prognosis of glioma patients (OS: HR $=0.67,95 \%$ Cl: 0.56-0.81, p<0.001; PFS: HR =0.81, 95\% Cl: 0.67-0.97, p = 0.025), compared with the no chemotherapy treatment. The Kaplan Meier survival curve described the survival rates of glioma patients with extent of resection (Fig. 1) and chemotherapy (Fig. 2) treatments, respectively. However, no significant associations were found between the age, gender, WHO grade, radiotherapy and the prognosis of glioma patients as measured by OS and PFS. 
Table 5

Univariate analysis of the impact of clinical factors and PVT1 polymorphisms on glioma patient OS and PFS

\begin{tabular}{|c|c|c|c|c|c|c|c|c|c|c|c|c|}
\hline \multirow[t]{2}{*}{ Variable } & \multirow[t]{2}{*}{ Classification } & \multirow{2}{*}{$\begin{array}{l}\text { No. of } \\
\text { patients/events }\end{array}$} & \multicolumn{5}{|l|}{ os } & \multirow{2}{*}{$\begin{array}{l}\text { No. of } \\
\text { patients/events }\end{array}$} & \multicolumn{4}{|l|}{ PFS } \\
\hline & & & $\begin{array}{l}1 \text { year } \\
\text { ST } \%\end{array}$ & $\begin{array}{l}\text { MST } \\
\text { (month) }\end{array}$ & $\begin{array}{l}\text { Log- } \\
\text { rank } \\
\text { p }\end{array}$ & $\begin{array}{l}\text { HR } \\
(95 \% \mathrm{Cl})\end{array}$ & $p$ & & $\begin{array}{l}1 \text { year } \\
\text { ST } \%\end{array}$ & $\begin{array}{l}\text { MST } \\
\text { (month) }\end{array}$ & $\begin{array}{l}\text { Log- } \\
\text { rank } \\
\text { p }\end{array}$ & $\begin{array}{l}\text { HF } \\
(9 !\end{array}$ \\
\hline \multirow[t]{2}{*}{ Gender } & Male & $321 / 284$ & 32.6 & 11 & & 1 & & $319 / 282$ & 20.3 & 8 & & 1 \\
\hline & Female & $257 / 230$ & 30.7 & 11 & 0.352 & $\begin{array}{l}1.08 \\
(0.91- \\
1.28)\end{array}$ & 0.394 & $254 / 228$ & 15.3 & 8 & 0.241 & $\begin{array}{l}1.1 \\
(0 . \\
1 . E\end{array}$ \\
\hline \multirow[t]{2}{*}{ Age(years) } & $<40$ & $258 / 221$ & 35.1 & 12 & & 1 & & $254 / 218$ & 20.2 & 8 & & 1 \\
\hline & $\geq 40$ & $320 / 293$ & 29.1 & 11 & 0.061 & $\begin{array}{l}1.17 \\
(0.98- \\
1.39)\end{array}$ & 0.086 & $319 / 292$ & 16.4 & 8 & 0.121 & $\begin{array}{l}1.1 \\
(0 . \\
1 . E\end{array}$ \\
\hline \multirow[t]{2}{*}{ WHO grade } & I & $371 / 324$ & 32.8 & 12 & & 1 & & $369 / 322$ & 19.1 & 8 & & 1 \\
\hline & ? & $207 / 190$ & 30.0 & 10 & 0.094 & $\begin{array}{l}1.15 \\
(0.96- \\
1.38)\end{array}$ & 0.125 & $204 / 188$ & 16.3 & 8 & 0.122 & $\begin{array}{l}1.1 \\
(0 . \\
1 . \Sigma\end{array}$ \\
\hline \multirow{2}{*}{$\begin{array}{l}\text { Extent of } \\
\text { resection }\end{array}$} & STR or NTR & $184 / 181$ & 19.6 & 12 & & 1 & & $181 / 178$ & 1.70 & 8 & & 1 \\
\hline & GTR & $394 / 333$ & 37.5 & 11 & $<.001$ & $\begin{array}{l}0.63 \\
(0.52- \\
0.76)\end{array}$ & $<.001$ & $392 / 332$ & 25.8 & 8 & $\begin{array}{l}<.001 \\
0.001\end{array}$ & $\begin{array}{l}0.5 \\
(0 . \\
0 . \overline{1}\end{array}$ \\
\hline \multirow[t]{3}{*}{ Radiotherapy } & No & $57 / 46$ & 43.9 & 12 & & 1 & & $54 / 43$ & 20.4 & 10 & & 1 \\
\hline & CRT & $156 / 128$ & 24.0 & 10 & & & & $155 / 127$ & 21.5 & 8 & & \\
\hline & Gamma knife & $365 / 340$ & 33.2 & 11 & 0.523 & $\begin{array}{l}1.07 \\
(0.94- \\
1.22)\end{array}$ & 0.314 & $364 / 340$ & 16.5 & 8 & 0.096 & $\begin{array}{l}1 . C \\
(0 . \\
1.2\end{array}$ \\
\hline \multirow[t]{2}{*}{ Chemotherapy } & No & $341 / 319$ & 27.0 & 9 & & 1 & & $340 / 318$ & 16.8 & 7 & & 1 \\
\hline & Yes & $237 / 195$ & 38.7 & 12 & $\begin{array}{l}<.001 \\
0.001\end{array}$ & $\begin{array}{l}0.67 \\
(0.56- \\
0.81)\end{array}$ & $\begin{array}{l}< \\
0.001\end{array}$ & 233/192 & 20.1 & 8 & 0.012 & $\begin{array}{l}0 . \varepsilon \\
(0 . \\
0 . c\end{array}$ \\
\hline \multirow[t]{3}{*}{ rs4410871 } & $\mathrm{CC}$ & $250 / 221$ & 34.7 & 11 & & 1 & & $248 / 219$ & 18.7 & 8 & & 1 \\
\hline & CT & $250 / 225$ & 30.8 & 11 & & $\begin{array}{l}0.98 \\
(0.75- \\
1.29)\end{array}$ & 0.905 & $247 / 223$ & 17.8 & 8 & & $\begin{array}{l}0 . \mathrm{c} \\
(0 . \\
1.2\end{array}$ \\
\hline & TT & $78 / 68$ & 25.6 & 11 & 0.809 & $\begin{array}{l}1.05 \\
(0.87- \\
1.26)\end{array}$ & 0.611 & $78 / 68$ & 17.0 & 8 & 0.899 & $\begin{array}{l}1 . C \\
(0 . \\
1.2\end{array}$ \\
\hline \multirow[t]{3}{*}{ rs4733789 } & $\mathrm{CC}$ & $181 / 163$ & 28.7 & 11 & & 1 & & $180 / 162$ & 17.4 & 8 & & 1 \\
\hline & $\mathrm{CT}$ & $285 / 249$ & 33.9 & 11 & & $\begin{array}{l}0.99 \\
(0.78- \\
1.27)\end{array}$ & 0.953 & $282 / 247$ & 20.4 & 8 & & $\begin{array}{l}0 . \mathrm{c} \\
(0 . \\
1.2\end{array}$ \\
\hline & TT & $112 / 102$ & 31.3 & 12 & 0.763 & $\begin{array}{l}0.94 \\
(0.77- \\
1.15)\end{array}$ & 0.537 & $111 / 101$ & 13.5 & 8 & 0.588 & $\begin{array}{l}0 . \mathrm{C} \\
(0 . \\
1.1\end{array}$ \\
\hline \multirow[t]{3}{*}{ rs13255292 } & $\mathrm{CC}$ & $365 / 325$ & 30.4 & 11 & & 1 & & $361 / 321$ & 17.7 & 8 & & 1 \\
\hline & CT & $195 / 171$ & 34.1 & 12 & & $\begin{array}{l}1.16 \\
(0.71- \\
1.89)\end{array}$ & 0.555 & $194 / 171$ & 18.4 & 8 & & $\begin{array}{l}1.1 \\
(0 . \\
1 . \varepsilon\end{array}$ \\
\hline & TT & $17 / 17$ & 29.4 & 10 & 0.333 & $\begin{array}{l}0.90 \\
(0.75- \\
1.08)\end{array}$ & 0.257 & $17 / 17$ & 17.6 & 8 & 0.407 & $\begin{array}{l}0 . c \\
(0 . \\
1 . C\end{array}$ \\
\hline
\end{tabular}

WHO: World Health Organization; GTR: gross-total resection; NTR: near-total resection; STR: sub-total resection; OS: overall survival; PFS: progression free sur survival rate; MST: median survival time

HR: hazard ratio; $95 \% \mathrm{Cl}$ : 95\% confidence interval

$\mathrm{P}<0.05$ indicates statistical significance. 


\section{PVT1 polymorphisms and prognosis of glioma patients}

We used the log-rank tests, Cox regression analysis (univariate and multivariate) and Kaplan Meier analysis to evaluate the effect of the four PVT1 polymorphisms on the glioma patients with OS and PFS (Table 5 and Table 6). However, there were no significant associations were found between the polymorphisms of PVT1 and the prognosis of glioma patients.

Table 6

Univariate analysis of the association between and glioma patient OS and PFS

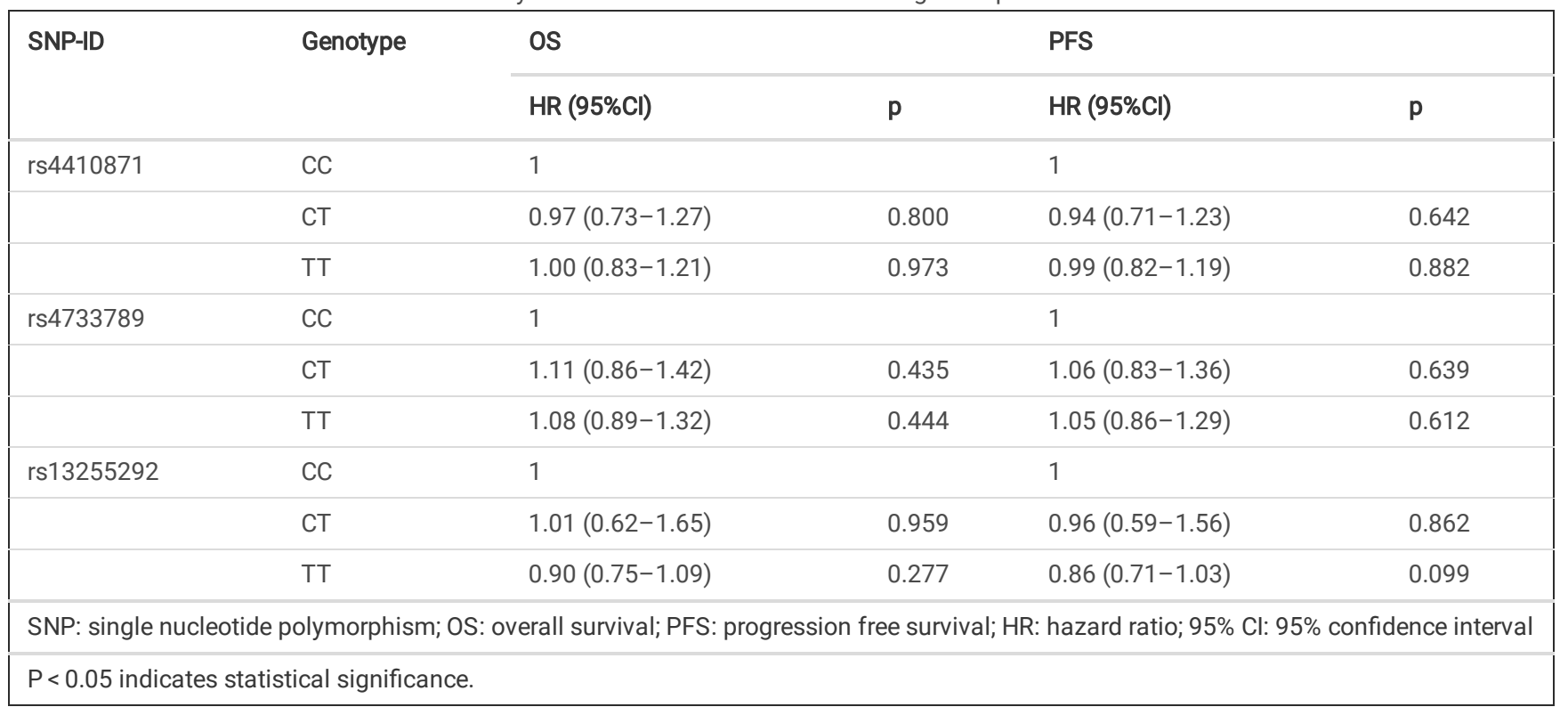

\section{Discussion}

To our knowledge, this case-control study is firstly to investigate the role of PVT1 polymorphisms (rs4410871, rs4733789 and rs13255292) in the susceptibility and survival of glioma in the Chinese Han population. We found that rs13255292 was associated with a decreased risk of glioma in the recessive model in overall or male; rs 4410871 was significantly associated with an increased the risk of glioma in age $\leq 40$ years old or female. Moreover, the extent of resection and chemotherapy were found to be key prognostic factors in survival of glioma patients. However, no effects were found between PVT1 polymorphisms on prognosis of glioma patients.

PVT1 is one of the transcribed IncRNAs located at the 8q24 human chromosomal region susceptibility locus. LncRNAs are key mediators of pathways involved in tumor suppression and oncogenesis affecting important cellular processes, such as chromatin reprogramming, cis- and trans-regulation of gene expression and mRNA processing [16-18]. PVT1 located downstream of MYC, has been proven to play an important role in cancer, and PVT1 dependence in cancer with MYC copy-number increase [19]. The upregulation of PVT1 has been found to be involved in poor prognosis in colorectal cancer [20], gastric cancer [21], pancreatic cancer [22], glioma [23]. PVT1 modulated GREM1 and BMP downstream signaling proteins through sponging miR-128-3p, thereby promoting tumorigenesis and progression of glioma [24]. The overexpression of PVT1 in glioma tissue and cells and promotes glioma cell proliferation and invasion by targeting miR-200a [25]. PVT1 knockdown could negatively regulate miR-424 to inhibit human glioma cell activity, migration and invasiveness [26].

The rs4410871 and rs13255292 in PVT1 were found to be significantly associated with the risk of glioma in the Chinese Han population. However, no association was found between rs 4733789 and glioma risk. These PVT1 polymorphisms have been reported to be associated with disease susceptibility. A Meta-analysis of genome-wide association studies (GWAS) identified that rs4410871 influenced allergic sensitization [27]. The rs4410871 was found to be associated with susceptibility to multiple sclerosis [28, 29]. Previous meta-analysis of GWAS identified that rs4733789 was associated with adult height in an East Asian population [30]. The men with the minor allele T in rs4476972 and with the major allele $\mathrm{C}$ in rs4733789 tend to have a lower risk of developing prostate cancer in African Americans population [31]. A GWAS and a pooled study of three Eastern Asian populations observed a significant association between rs13255292 (PVT1) and diffuse large B-cell lymphoma risk [32, 33]. The rs13255292 was also found to be associated with ovarian cancer [34]. However, this study was firstly to investigate the association between the three PVT1 polymorphisms and glioma risk. Therefore, the results need to be confirmed in multi-ethnic population with larger scale.

This study also explored the impact of clinical features and PVT1 polymorphisms on the prognosis of glioma patients. Our results found that extent of resection and chemotherapy were key prognostic factors in survival of glioma patients. However, gender, age, who grade, radiotherapy, and PVT1 polymorphisms have no effects on survival of glioma patients. Wang et al. indicated that degree of resection and pathological grade were independent prognostic factors for patients with malignant gliomas, but age and gender have no effect on glioma survival outcome [35]. The extent of resection and sex was found to be as prognostic factors for overall survival in pediatric high-grade glioma [36]. This comprehensive analysis of multicentric glioma patients revealed that age $>54$ years old, surgical resection, and radiotherapy were significantly associated with improved survival and were independent prognostic factors for OS. Radiotherapy and radiotherapy combined with chemotherapy were independent prognostic factors for surgical patients' OS as well [37]. There 
are many reasons for conflicting results, such as differences in sample size, ethnicity, tumor grade, and experiment method. Therefore, further study is needed to confirm the results.

There were several limitations in the present study. First, only three SNPs in PVT1 were selected in this study which may not represent a comprehensive view of PVT1 variation. Further studies on variation in susceptible regions of PVT1 are needed. Second, data were not available for some risk factors (e.g., cigarette smoking, alcohol consumption), therefore, these factors should be taken into account in future studies. Third, since this is a very preliminary study, further functional studies are also required to explore the mechanisms of the PVT1 polymorphisms affected the risk and prognosis in glioma patient.

\section{Conclusions}

In conclusion, the results indicated that PVT1 polymorphisms (rs13255292 and rs4410871) were significantly associated with the risk of glioma. The extent of resection and chemotherapy were found to be key prognostic factors in survival of glioma patients. However, the PVT1 polymorphisms have no effects on prognosis of glioma patients. Further study with large samples on the role of PVT1 polymorphisms in the susceptibility and survival of glioma is warranted to obtain more conclusive outcomes.

\section{Declarations}

\section{Ethics approval and consent to participate}

The study protocol was approved by the Clinical Research Ethics Committee of the Tangdu Hospital of The Fourth Military Medical University, and was conducted in accordance with the principles of the Declaration of Helsinki. Blood samples and signed informed consent forms were obtained from enrolled individuals prior to their participation in the study.

\section{Consent for publication}

Not applicable.

\section{Competing interests}

The authors have declared that no competing interest exists.

\section{Funding}

None.

\section{Authors' contributions}

Ya Gao participated in the study design; Xiaoying Ding drafted the manuscript; Yaqin Zhao was involved with the analysis of data; Haozheng Yuan and Yong Zhang performed the experiments. All authors have read and approved the final manuscript.

\section{Acknowledgements}

We are grateful to the patients and control individuals for their participation in the study. We also thank the clinicians and hospital staff who contributed to sample and data collection for this study.

\section{References}

1. Reni M, Mazza E, Zanon S, Gatta G, Vecht CJ. Central nervous system gliomas. Crit Rev Oncol Hematol. 2017;113:213-34; doi: 10.1016/j.critrevonc.2017.03.021.

2. Bray F, Ferlay J, Soerjomataram I, Siegel RL, Torre LA, Jemal A. Global cancer statistics 2018: GLOBOCAN estimates of incidence and mortality worldwide for 36 cancers in 185 countries. CA Cancer J Clin. 2018;68 6:394-424; doi: 10.3322/caac.21492.

3. Chen W, Zheng R, Baade PD, Zhang S, Zeng H, Bray F, et al. Cancer statistics in China, 2015. CA Cancer J Clin. 2016;66 2:115-32; doi: $10.3322 /$ caac. 21338 .

4. Ohgaki H, Kleihues P. Epidemiology and etiology of gliomas. Acta neuropathologica. 2005;109 1:93-108; doi: 10.1007/s00401-005-0991-y.

5. Hayashi S, Kitamura Y, Hirose Y, Yoshida K, Sasaki H. Molecular-genetic and clinicopathological prognostic factors in patients with gliomas showing total 1 19 19 loss: gain of chromosome 19p and histological grade III negatively correlate with patient's prognosis. Journal of neuro-oncology. 2017;132 1:11926; doi: 10.1007/s11060-016-2344-1.

6. Guo X, Song J, Zhao J, Wang B, Yang Z, Sun P, et al. Impact of ANXA5 polymorphisms on glioma risk and patient prognosis. Journal of neuro-oncology. 2019;142 1:11-26; doi: 10.1007/s11060-018-03069-9.

7. Li G, Zhang Z, Tu Y, Jin T, Liang H, Cui G, et al. Correlation of microRNA-372 upregulation with poor prognosis in human glioma. Diagnostic pathology. 2013;8:1; doi: 10.1186/1746-1596-8-1.

8. Du SL, Geng TT, Feng T, Chen CP, Jin TB, Chen C. The RTEL1 rs6010620 polymorphism and glioma risk: a meta-analysis based on 12 case-control studies. Asian Pacific journal of cancer prevention : APJCP. 2014;15 23:10175-9. 
9. Jin TB, Du S, Zhu XK, Li G, Ouyang Y, He N, et al. Polymorphism in the IL4R gene and clinical features are associated with glioma prognosis: Analyses of case-cohort studies. Medicine. 2016;95 31:e4231; doi: 10.1097/MD.0000000000004231.

10. Colombo T, Farina L, Macino G, Paci P. PVT1: a rising star among oncogenic long noncoding RNAs. BioMed research international. 2015;2015:304208; doi: 10.1155/2015/304208.

11. Zou H, Wu LX, Yang Y, Li S, Mei Y, Liu YB, et al. IncRNAs PVT1 and HAR1A are prognosis biomarkers and indicate therapy outcome for diffuse glioma patients. Oncotarget. 2017;8 45:78767-80; doi: 10.18632/oncotarget.20226.

12. Ma Y, Wang P, Xue Y, Qu C, Zheng J, Liu X, et al. PVT1 affects growth of glioma microvascular endothelial cells by negatively regulating miR-186. Tumour biology : the journal of the International Society for Oncodevelopmental Biology and Medicine. 2017;39 3:1010428317694326; doi: $10.1177 / 1010428317694326$.

13. Moschovis D, Vasilaki E, Tzouvala M, Karamanolis G, Katifelis H, Legaki E, et al. Association between genetic polymorphisms in long non-coding RNAs and pancreatic cancer risk. Cancer biomarkers : section A of Disease markers. 2019;24 1:117-23; doi: 10.3233/CBM-181959.

14. Zhang Z, Zhu Z, Zhang B, Li W, Li X, Wu X, et al. Frequent mutation of rs13281615 and its association with PVT1 expression and cell proliferation in breast cancer. Journal of genetics and genomics = Yi chuan xue bao. 2014;41 4:187-95; doi: 10.1016/j.jgg.2014.03.006.

15. Zhang W, Xiao J, Lu X, Liu T, Jin X, Xiao Y, et al. PVT1 (rs13281615) and miR-146a (rs2910164) polymorphisms affect the prognosis of colon cancer by regulating COX2 expression and cell apoptosis. Journal of cellular physiology. 2019; doi: 10.1002/jcp.28377.

16. Gibb EA, Brown CJ, Lam WL. The functional role of long non-coding RNA in human carcinomas. Molecular cancer. 2011;10:38; doi: 10.1186/1476-459810-38.

17. Zeng S, Xiao YF, Tang B, Hu CJ, Xie R, Yang SM, et al. Long Noncoding RNA in Digestive Tract Cancers: Function, Mechanism, and Potential Biomarker. The oncologist. 2015;20 8:898-906; doi: 10.1634/theoncologist.2014-0475.

18. Guttman M, Rinn JL. Modular regulatory principles of large non-coding RNAs. Nature. 2012;482 7385:339-46; doi: 10.1038/nature10887.

19. Tseng YY, Moriarity BS, Gong W, Akiyama R, Tiwari A, Kawakami H, et al. PVT1 dependence in cancer with MYC copy-number increase. Nature. 2014;512 7512:82-6; doi: 10.1038/nature13311.

20. Takahashi Y, Sawada G, Kurashige J, Uchi R, Matsumura T, Ueo H, et al. Amplification of PVT-1 is involved in poor prognosis via apoptosis inhibition in colorectal cancers. British journal of cancer. 2014;110 1:164-71; doi: 10.1038/bjc.2013.698.

21. Kong R, Zhang EB, Yin DD, You LH, Xu TP, Chen WM, et al. Long noncoding RNA PVT1 indicates a poor prognosis of gastric cancer and promotes cell proliferation through epigenetically regulating p15 and p16. Molecular cancer. 2015;14:82; doi: 10.1186/s12943-015-0355-8.

22. Huang C, Yu W, Wang Q, Cui H, Wang Y, Zhang L, et al. Increased expression of the IncRNA PVT1 is associated with poor prognosis in pancreatic cancer patients. Minerva medica. 2015;106 3:143-9.

23. Yang A, Wang H, Yang X. Long non-coding RNA PVT1 indicates a poor prognosis of glioma and promotes cell proliferation and invasion via target EZH2. Bioscience reports. 2017;37 6; doi: 10.1042/BSR20170871.

24. Fu C, Li D, Zhang X, Liu N, Chi G, Jin X. LncRNA PVT1 Facilitates Tumorigenesis and Progression of Glioma via Regulation of MiR-128-3p/GREM1 Axis and BMP Signaling Pathway. Neurotherapeutics : the journal of the American Society for Experimental NeuroTherapeutics. 2018;15 4:1139-57; doi: 10.1007/s13311-018-0649-9.

25. Zhang Y, Yang G, Luo Y. Long non-coding RNA PVT1 promotes glioma cell proliferation and invasion by targeting miR-200a. Experimental and therapeutic medicine. 2019;17 2:1337-45; doi: 10.3892/etm.2018.7083.

26. Han Y, Li X, Yan J, Ma C, Zheng X, Zhang J, et al. Knockdown of LncRNA PVT1 inhibits glioma progression by regulating miR-424 expression. Oncology research. 2019; doi: 10.3727/096504018X15424939990246.

27. Bonnelykke K, Matheson MC, Pers TH, Granell R, Strachan DP, Alves AC, et al. Meta-analysis of genome-wide association studies identifies ten loci influencing allergic sensitization. Nature genetics. 2013;45 8:902-6; doi: 10.1038/ng.2694.

28. Gourraud PA, Harbo HF, Hauser SL, Baranzini SE. The genetics of multiple sclerosis: an up-to-date review. Immunological reviews. 2012;248 1:87-103; doi: 10.1111/j.1600-065X.2012.01134.x.

29. International Multiple Sclerosis Genetics C, Beecham AH, Patsopoulos NA, Xifara DK, Davis MF, Kemppinen A, et al. Analysis of immune-related loci identifies 48 new susceptibility variants for multiple sclerosis. Nature genetics. 2013;45 11:1353-60; doi: 10.1038/ng.2770.

30. He M, Xu M, Zhang B, Liang J, Chen P, Lee JY, et al. Meta-analysis of genome-wide association studies of adult height in East Asians identifies 17 novel loci. Human molecular genetics. 2015;24 6:1791-800; doi: 10.1093/hmg/ddu583.

31. Lin HY, Callan CY, Fang Z, Tung HY, Park JY. Interactions of PVT1 and CASC11 on Prostate Cancer Risk in African Americans. Cancer epidemiology, biomarkers \& prevention : a publication of the American Association for Cancer Research, cosponsored by the American Society of Preventive Oncology. 2019; doi: 10.1158/1055-9965.EPI-18-1092.

32. Cerhan JR, Berndt SI, Vijai J, Ghesquieres H, McKay J, Wang SS, et al. Genome-wide association study identifies multiple susceptibility loci for diffuse large B cell lymphoma. Nature genetics. 2014;46 11:1233-8; doi: 10.1038/ng.3105.

33. Bassig BA, Cerhan JR, Au WY, Kim HN, Sangrajrang S, Hu W, et al. Genetic susceptibility to diffuse large B-cell lymphoma in a pooled study of three Eastern Asian populations. European journal of haematology. 2015;95 5:442-8; doi: 10.1111/ejh.12513.

34. Kim S, Wang M, Tyrer JP, Jensen A, Wiensch A, Liu G, et al. A comprehensive gene-environment interaction analysis in Ovarian Cancer using genome-wide significant common variants. International journal of cancer. 2019;144 9:2192-205; doi: 10.1002/ijc.32029.

35. Wang HW, Xu ZK, Song Y, Liu YG. Correlations of MGMT genetic polymorphisms with temozolomide resistance and prognosis of patients with malignant gliomas: a population-based study in China. Cancer gene therapy. 2017;24 5:215-20; doi: 10.1038/cgt.2017.7.

Page $13 / 14$ 
36. McCrea HJ, Bander ED, Venn RA, Reiner AS, Iorgulescu JB, Puchi LA, et al. Sex, Age, Anatomic Location, and Extent of Resection Influence Outcomes in Children With High-grade Glioma. Neurosurgery. 2015;77 3:443-52; discussion 52-3; doi: 10.1227/NEU.0000000000000845.

37. Wang T, Niu X, Gao T, Zhao L, Li J, Gan Y, et al. Prognostic Factors for Survival Outcome of High-Grade Multicentric Glioma. World Neurosurg. 2018;112:e269-e77; doi: 10.1016/j.wneu.2018.01.035.

\section{Figures}
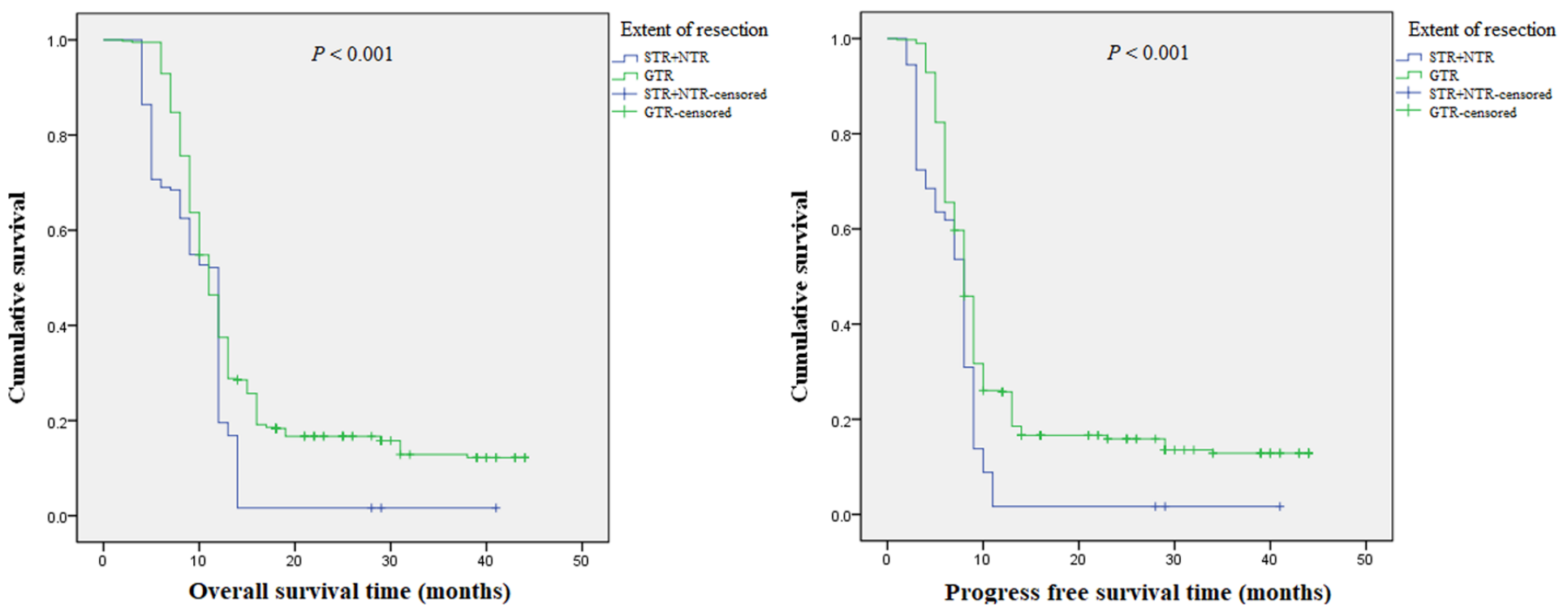

Figure 1

Kaplan-Meier curves for overall survival and progression-free survival of extent of resection in glioma patients
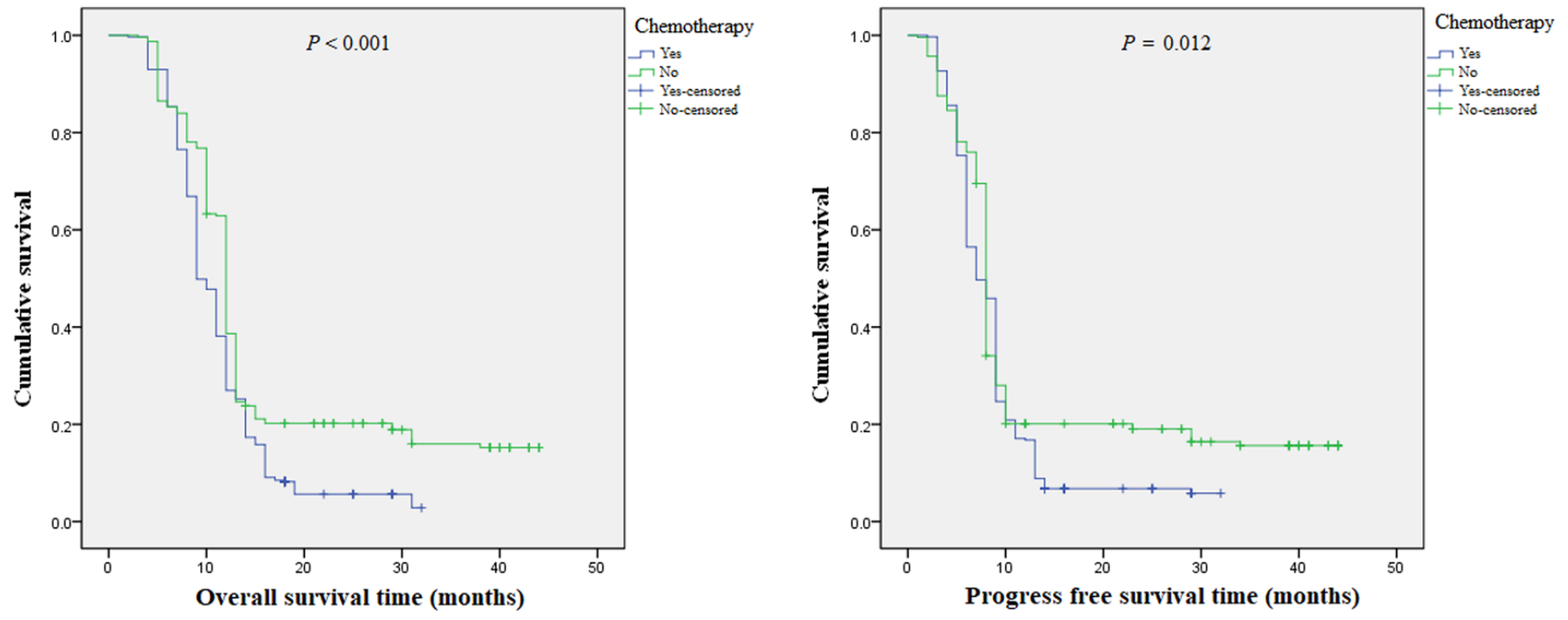

Figure 2

Kaplan-Meier curves for overall survival and progression-free survival of chemotherapy in glioma patients 